\title{
The Nme gene family in fish
}

\author{
T. Desvignes $^{a, b}$, A. Fostier ${ }^{a}$, C. Fauvel ${ }^{b}$, J. Bobe $^{a,{ }^{*}}$ \\ ${ }^{a}$ Fish Physiology and Genomics, INRA, UR1037 LPGP, Campus de Beaulieu, 35042, Rennes, France \\ b IFREMER, LALR, 34250, Palavas Les Flots, France \\ *: Corresponding author : Julien Bobe, email address : Julien.Bobe@rennes.inra.fr
}

\begin{abstract}
:
The Nme gene family, also known as Nm23 or NDPK, is a very ancient gene family that can be found in all kingdoms of life. In the late eighties, a gene of the Nme family, NME1, was identified as the first metastatic suppressor gene, resulting in a major interest for this family. Due to the complexity of the family, the need for a unified and evolutionary-supported gene nomenclature was recently stressed by the scientific community. Based on a complete evolutionary history study of the gene family in metazoans and vertebrates, a unified nomenclature was recently proposed and accepted by gene nomenclature consortia. In addition to its well-documented role in tumor metastasis, members of the Nme family are also involved in a wide variety of cellular and physiological processes. Available data in non-mammalian species remain, however, scarce with the noticeable exception of Drosophila in which a major role in development was reported. In fish, very few studies have specifically investigated the role of $n m e$ genes. Several transcriptomic and proteomic studies have, however, revealed the expression of $n m e$ genes in various fish organs and tissues, in mature oocytes, and during embryonic development. Altogether, interest for the Nme gene family in fish is growing and new functions/roles in fish biology are expected to be discovered in the forthcoming years. Here, we briefly review the current knowledge of the Nme family in fish.
\end{abstract}

Keywords: Nm23 ; NDPK ; RP2 ; Teleost ; Oocyte ; Ovary 


\section{Introduction}

The Nme gene family, also called Nm23 or NDPK, is a very ancient gene family found in all kingdoms of life, i.e. eubacteria (Hama et al. 1991; Lu et al. 1995), archaea (Polosina et al. 1998) and eukaryotes (Desvignes et al. 2010). In the late eighties, a gene of the Nme family, $N M E 1$, was identified as the first metastatic suppressor gene (Steeg et al. 1988). Nme genes have however been shown to be involved in multiple physiological and pathological processes such as cellular differentiation, embryonic development, metastatic dissemination, and cilia functions (Biggs et al. 1990; Boissan et al. 2009). In eukaryotes, Nme proteins are separated in 2 different groups - group I and group II - based on their nucleoside diphosphate kinase (NDPK) activity and their evolutionary history (Boissan et al. 2009; Desvignes et al. 2009; Desvignes et al. 2010) (Fig. 1 and 2). The biochemical NDPK activity, that has been studied for group I members and Nme6 protein (Tsuiki et al. 1999; Boissan et al. 2009; Perina et al. 2011), has however never been studied in fish. Nevertheless, sequence analysis of zebrafish Nme proteins, based on identification of amino acid supposed to be crucial for NDPK function, suggests conservation of NDPK activity in (co-)orthologous zebrafish proteins (Desvignes et al. 2009). While genes of the group II are well conserved among metazoans, genes of the group I underwent several independent duplications. In non-vertebrate metazoan species, the group I ancestor gene was independently duplicated in most lineages (Desvignes et al. 2010). In the vertebrate radiation, the number of group I genes is higher than in other metazoans and orthology relationships more difficult to analyze because of the successive whole genome duplication events that occurred early in the vertebrate radiation and in the teleost lineage (Postlethwait et al. 1998; Dehal and Boore 2005; Desvignes et al. 2009). The most recently identified vertebrate-specific Nme gene, Nme10 also known as $R P 2$, has a different evolutionary history and the corresponding amino acid sequence results from the fusion of a partial NDPk domain into a tubulin binding protein (Desvignes et al. 2009). In teleosts, 9 nme genes can be found (Fig. 1) with some species-specific differences such as additional copies due to additional whole genome duplication (i.e. in salmonids), gene loss (e.g. nme $2 b$ in stickleback), or lineage-specific duplications (e.g. duplicated $n m e 2 b$ in zebrafish). 


\section{A recently normalized gene nomenclature}

In order to study nme genes in teleost species, it is important to know precisely the identity of each gene and how a specific gene relates to homologs in other metazoan species. It is indeed important to know whether or not a specific gene has a clear (and possibly unique) ortholog in other species. In 2009, the scientific community stressed the need for a unified Nme gene nomenclature (Mehta and Orchard 2009). All Nme genes had previously been named without any consistency among species or within each species. In most cases, the name was only based on the name of the mammalian sequence showing the highest percentage of identity regardless of the evolutionary history of the genes. Because of high sequence identity shared by Nme genes in metazoans, this could greatly complicate interpretation of already published data or, in some cases, lead to misinterpretation. For instance, the zebrafish nme2bl gene had been called NM23$B$ (Lee and Lee 2000), NM23-Z1 (Bilitou et al. 2009) and NDPK B (Hippe et al. 2009) while the zebrafish $n m e 2 b 2$ had been called $N D K-Z 2$ (Masuda et al. 2009) and NDPK A (Hippe et al. 2009). Using NDPK $A$ and NDPK $B$ when referring to $n m e 2 b 2$ and $n m e 2 b 1$ zebrafish genes obviously leads the reader to consider a oneto-one orthology relationship with mammalian NME1 (previously called NDPK $A$ ) and NME2 (previously called $N D P K B$ ). In fact, the evolutionary history of the vertebrate Nme gene family clearly demonstrated that the two mammalian genes NME1 and NME2 were co-orthologs of the three zebrafish genes nme $2 a, n m e 2 b 1$ and nme $2 b 2$ as the duplication events that gave rise to multiple genes in the tetrapod and the teleost radiations are independent (Desvignes et al. 2009) (Fig. 2). Based on the evolutionary history analysis of the whole Nme gene family in metazoans (Desvignes et al. 2010) and vertebrates (Desvignes et al. 2009), a unified nomenclature was proposed. The proposed nomenclature is based on the official nomenclature in use in mammals, the official rules for gene nomenclature, and the evolutionary history of the gene family. All genes and proteins were thus named using the official prefix "Nme" referring to "Non-Metastatic cells, Expressed in" and numbered according to their orthology relationship with their mammalian orthologs. No indication concerning the species (e.g., "Z" for zebrafish) was included in the gene name as recommended by international 
committees for gene nomenclature such as HUGO ( 2009), MGI ( 2012a) or ZFIN ( 2012b) (Fig. 1). This nomenclature is now used by ZFIN.

\section{A growing interest for nme genes in fish}

Despite the known importance of $N M E$ genes in several human pathologies and their common use as clinical markers of tumor aggressiveness (Shoushtari et al. 2011), their role in non-pathological physiological processes has, in contrast, received far less attention. Many researches are conducted in zebrafish with the objective of better understanding biological processes related to human diseases because of better shared organ architecture than drosophila or nematode worms and easier use in laboratory than are mice or rats (Ingham 2009). Furthermore, the Teleost Genome Duplication (TGD or 3R) has in some cases led to gene subfunctionalization that can facilitate the analysis of a specific function. In some cases however this duplication event has led to gene neo-functionalization or loss that can make transfer of knowledge between species more problematic. As shown on Figure 2, independent duplication events have led to three nme2 genes in zebrafish and two in mammals. In this context, it is thus difficult to directly infer a specific function from zebrafish to humans. Nevertheless, several recent articles demonstrated an important role of nme genes in fish biology that might be beneficial for biomedical researchers to decipher the role of $N M E$ genes in human pathologies such as cardiomyopathy (Hippe et al. 2009; Hippe et al. 2011) or XLinked Retinitis Pigmentosa (Hurd et al. 2010; Shu et al. 2011; Patil et al. 2011).

As indicated above, several transcriptomic and proteomic analyses led to the identification of Nme genes and proteins in various fish organs and tissues. For instance, the Ndpk activity has been used in several studies as an indicator of protein synthesis in fish muscle (Couture et al. 1998; Morbey et al. 2010). Indeed, expression survey of the whole gene family in zebrafish has revealed that one nme gene, nme2b2, was highly and predominantly expressed in muscle (Desvignes et al. 2009). Some other nme family members, known to be restricted to the testis in tetrapods, i.e. nme 5 and nme8, are also specifically expressed in the testis of teleost fish (Desvignes et al. 2009).

Interestingly, several independent genomic studies conducted on various fish species identified various Nme transcripts and/or proteins as expressed in the 
oocyte or during early embryonic development (Murphy et al. 2000; Bai et al. 2007; Crespel A et al. 2008; Ziv et al. 2008; Keyvanshokooh and Vaziri 2008; Hurd et al. 2010; Desvignes et al. 2011; Shu et al. 2011). However, the corresponding expression profiles or patterns were not investigated in these studies. In sea bass eggs (Dicentrarchus labrax), it was shown that a Nme2related protein was differentially expressed between low and good quality egg batches (Crespel A et al. 2008). This observation is of particular interest in the light of the existing literature concerning drosophila orthologous gene awd which is involved in oogenesis and embryonic wing development, as well as one mammalian co-orthologous genes that has been shown to be involved in embryonic stem cell differentiation by regulating $c-m y c$ expression (Postel et al. 1993; Thakur et al. 2009; Zhu et al. 2009). Nme2-related protein might therefore be required for embryonic stem cell differentiation in fish early embryonic development. Another gene, nme3, known to be involved in neural development of the mouse (Amrein et al. 2005), was shown to be maternally-inherited in the mature oocyte / early embryo and also expressed in the developing neural system of zebrafish embryos (Desvignes et al. 2011). Similarly, nme6 - a group II nme gene - was reported as maternally-inherited but exhibited a sharp decrease at fertilization (Desvignes et al. 2011). The role of this maternal mRNA in early embryonic development remains however to be elucidated. Recently, two articles showed an expression of the nme10/RP2 gene in the fish oocyte (Hurd et al. 2010; Shu et al. 2011) but the expression level was not quantified and the role of the maternally inherited transcripts was not investigated. Nevertheless, these authors demonstrated a compulsory role of $n m e 10 / R P 2$ in zebrafish development as knock-down of the protein resulted in high developmental defects related to primary cilia troubles.

\section{Conclusion}

The Nme gene family is shared by all metazoan species and is involved in a wide variety of physiological processes. The strikingly conserved gene and protein sequence features that can be observed within the animal kingdom for some Nme genes suggest a participation in key biological processes that could play an important role in fish, including during oocyte and embryo development. The recent development of a unified gene nomenclature sets grounds for in depth 
analysis of the Nme gene family in several physiological functions, including reproduction.

\section{Acknowledgements}

This work was supported by the European Community's Seventh Framework Program (FP7/2007-2013) under grant agreement no. 222719 - LIFECYCLE.

\section{References}

\section{Legends}

Fig.1 Nme genes and proteins in teleost fish. Zebrafish Nme protein structure and size are given. Gene duplication events and tissular expression relate to public sequences analysis and available literature. 4R refers to the Salmonid-specific Genome Duplication.

Fig.2 Schematic vertebrate Nme genes evolution. Adapted from Desvignes et al (2009). 1R and 3R whole genome duplication events that gave rise to duplicated genes are shown. Stars indicate cis-duplication events.

\section{Reference List}

2009. HUGO Gene Nomenclature Committee at the European Bioinformatics Institute. HUGO

2012a. MGI Mouse genome Informatics. MGI

2012b. ZFIN The Zebrafish Model Organism Database. ZFIN

Amrein, L., Barraud, P., Daniel, J.Y., Perel, Y. and Landry, M. 2005. Expression patterns of nm23 genes during mouse organogenesis. Cell Tissue Res 322: 365-378.

Bai, J.L., Solberg, C., Fernandes, J.M.O. and Johnston, I.A. 2007. Profiling of maternal and developmental-stage specific mRNA transcripts in Atlantic halibut Hippoglossus hippoglossus. Gene 386: 202-210.

Biggs, J., Hersperger, E., Steeg, P.S., Liotta, L.A. and Shearn, A. 1990. A Drosophila gene that is homologous to a mammalian gene associated with tumor metastasis codes for a nucleoside diphosphate kinase. Cell 63: 933-940.

Bilitou, A., Watson, J., Gartner, A. and Ohnuma, S.I. 2009. The NM23 family in development. Mol Cell Biochem 
Boissan, M., Dabernat, S., Peuchant, E., Schlattner, U., Lascu, I. and Lacombe, M.L. 2009. The mammalian Nm23/NDPK family: from metastasis control to cilia movement. Mol Cell Biochem 329: 51-62.

Couture, P., Dutil, J.D. and Guderley, H. 1998. Biochemical correlates of growth and condition in juvenile Atlantic cod (Gadus morhua) from Newfoundland. Canadian Journal of Fisheries and Aquatic Sciences 55: 1591-1598.

Crespel A, Rime H, Fraboulet E, Bobe J and Fauvel C 2008. Egg quality in domesticated and wild seabass (Dicentrarchus labrax): A proteomic analysis. Cybium 32(2) suppl.: 205-

Dehal, P., Boore, J.L. 2005. Two Rounds of Whole Genome Duplication in the Ancestral Vertebrate. PLoS Biol 3: e314-

Desvignes, T., Fauvel, C. and Bobe, J. 2011. The nme gene family in zebrafish oogenesis and early development. Naunyn-Schmiedeberg's Archives of Pharmacology1-11.

Desvignes, T., Pontarotti, P. and Bobe, J. 2010. Nme Gene Family Evolutionary History Reveals Pre-Metazoan Origins and High Conservation between Humans and the Sea Anemone, Nematostella vectensis. PLoS ONE 5: e15506-

Desvignes, T., Pontarotti, P., Fauvel, C. and Bobe, J. 2009. Nme protein family evolutionary history, a vertebrate perspective. BMC Evolutionary Biology 9: 256-

Hama, H., Almaula, N., Lerner, C.G., Inouye, S. and Inouye, M. 1991. Nucleoside diphosphate kinase from Escherichia coli; its overproduction and sequence comparison with eukaryotic enzymes. Gene 105: 31-36.

Hippe, H.J., Wolf, N.M., Abu-Taha, I., Mehringer, R., Just, S., Lutz, S., Niroomand, F., Postel, E.H., Katus, H.A., Rottbauer, W. and Wieland, T. 2009. The interaction of nucleoside diphosphate kinase $\mathrm{B}$ with $\mathrm{G}+1+1$, dimers controls heterotrimeric $\mathrm{G}$ protein function. Proceedings of the National Academy of Sciences 106: 16269-16274.

Hippe, H.J., Wolf, N., Abu-Taha, H., Lutz, S., Le Lay, S., Just, S., Rottbauer, W., Katus, H. and Wieland, T. 2011. Nucleoside diphosphate kinase B is required for the formation of heterotrimeric $\mathrm{G}$ protein containing caveolae. Naunyn-Schmiedeberg's Archives of Pharmacology1-12.

Hurd, T., Zhou, W., Jenkins, P., Liu, C.J., Swaroop, A., Khanna, H., Martens, J., Hildebrandt, F. and Margolis, B. 2010. The retinitis pigmentosa protein RP2 interacts with polycystin 2 and regulates cilia-mediated vertebrate development. Human Molecular Genetics 19: 4330-4344.

Ingham, P.W. 2009. The power of the zebrafish for disease analysis. Hum. Mol Genet 18: R107R112.

Keyvanshokooh, S., Vaziri, B. 2008. Proteome analysis of Persian sturgeon (Acipenser persicus) ova. Animal Reproduction Science 109: 287-297.

Lee, J.S., Lee, S.H. 2000. Cloning and characterization of cDNA encoding zebrafish Danio rerio NM23-B gene. Gene 245: 75-79.

Lu, Q., Zhang, X., Almaula, N., Mathews, C.K. and Inouye, M. 1995. The gene for nucleoside diphosphate kinase functions as a mutator gene in Escherichia coli. J Mol Biol 254: 337 341.

Masuda, Y., Oku, H., Okumura, T., Nomura, K. and Kurokawa, T. 2009. Feeding restriction alters expression of some ATP related genes more sensitively than the RNA/DNA ratio in zebrafish, Danio rerio. Comparative Biochemistry and Physiology Part B: Biochemistry and Molecular Biology 152: 287-291. 
Mehta, A., Orchard, S. 2009. Nucleoside diphosphate kinase (NDPK, NM23, AWD): recent regulatory advances in endocytosis, metastasis, psoriasis, insulin release, fetal erythroid lineage and heart failure; translational medicine exemplified. Mol Cell Biochem 329: 315 .

Morbey, Y.E., Couture, P., Busby, P. and Shuter, B.J. 2010. Physiological correlates of seasonal growth patterns in lake trout Salvelinus namaycush. Journal of Fish Biology 77: 22982314.

Murphy, M., Harte, T., McInerney, J. and Smith, T.J. 2000. Molecular cloning of an Atlantic salmon nucleoside diphosphate kinase cDNA and its pattern of expression during embryogenesis. Gene 257: 139-148.

Patil, S.B., Hurd, T.W., Ghosh, A.K., Murga-Zamalloa, C.A. and Khanna, H. 2011. Functional Analysis of Retinitis Pigmentosa 2 (RP2) Protein Reveals Variable Pathogenic Potential of Disease-Associated Missense Variants. PLoS ONE 6: e21379-

Perina, D., Bosnar, M., Mikoc, A., Muller, W. and -Cetkovic, H. 2011. Characterization of Nme6like gene/protein from marine sponge Suberites domuncula. Naunyn-Schmiedeberg's Archives of Pharmacology1-10.

Polosina, Y.Y., Jarrell, K.F., Fedorov, O.V. and Kostyukova, A.S. 1998. Nucleoside diphosphate kinase from haloalkaliphilic archaeon Natronobacterium magadii: purification and characterization. Extremophiles 2: 333-338.

Postel, E.H., Berberich, S.J., Flint, S.J. and Ferrone, C.A. 1993. Human c-myc transcription factor $\mathrm{PuF}$ identified as nm23-H2 nucleoside diphosphate kinase, a candidate suppressor of tumor metastasis. Science 261: 478-480.

Postlethwait, J.H., Yan, Y.L., Gates, M.A., Horne, S., Amores, A., Brownlie, A., Donovan, A., Egan, E.S., Force, A., Gong, Z., Goutel, C., Fritz, A., Kelsh, R., Knapik, E., Liao, E., Paw, B., Ransom, D., Singer, A., Thomson, M., Abduljabbar, T.S., Yelick, P., Beier, D., Joly, J.S., Larhammar, D., Rosa, F., Westerfield, M., Zon, L.I., Johnson, S.L. and Talbot, W.S. 1998. Vertebrate genome evolution and the zebrafish gene map. Nat Genet 18: 345349.

Shoushtari, A.N., Szmulewitz, R.Z. and Rinker-Schaeffer, C.W. 2011. Metastasis-suppressor genes in clinical practice: lost in translation? Nat Rev Clin. Oncol. 8: 333-342.

Shu, X., Zeng, Z., Gautier, P., Lennon, A., Gakovic, M., Cheetham, M.E., Patton, E.E. and Wright, A.F. 2011. Knockdown of the Zebrafish Ortholog of the Retinitis Pigmentosa 2 (RP2) Gene Results in Retinal Degeneration. Investigative Ophthalmology \& Visual Science 52: 2960-2966.

Steeg, P.S., Bevilacqua, G., Kopper, L., Thorgeirsson, U.P., Talmadge, J.E., Liotta, L.A. and Sobel, M.E. 1988. Evidence for a Novel Gene Associated With Low Tumor Metastatic Potential. JNCI Journal of the National Cancer Institute 80: 200-204.

Thakur, R.K., Kumar, P., Halder, K., Verma, A., Kar, A., Parent, J.L., Basundra, R., Kumar, A. and Chowdhury, S. 2009. Metastases suppressor NM23-H2 interaction with Gquadruplex DNA within c-MYC promoter nuclease hypersensitive element induces cMYC expression. Nucleic Acids Research 37: 172-183.

Tsuiki, H., Nitta, M., Furuya, A., Hanai, N., Fujiwara, T., Inagaki, M., Kochi, M., Ushio, Y., Saya, H. and Nakamura, H. 1999. A novel human nucleoside diphosphate (NDP) kinase, Nm23-H6, localizes in mitochondria and affects cytokinesis. J Cell Biochem 76: 254-269.

Zhu, S., Wurdak, H., Wang, J., Lyssiotis, C.A., Peters, E.C., Cho, C.Y., Wu, X. and Schultz, P.G. 2009. A Small Molecule Primes Embryonic Stem Cells for Differentiation. Cell stem cell 4: 416-426. 
Ziv, T., Gattegno, T., Chapovetsky, V., Wolf, H., Barnea, E., Lubzens, E. and Admon, A. 2008. Comparative proteomics of the developing fish (zebrafish and gilthead seabream) oocytes. Comparative Biochemistry and Physiology Part D: Genomics and Proteomics 3: $12-35$. 


\begin{tabular}{|c|c|c|c|c|}
\hline & $\begin{array}{l}\text { Gene } \\
\text { Symbol }\end{array}$ & Protein structure (aa number) & Gene evolution in teleost & Main tissular expression \\
\hline \multirow{4}{*}{ 믈 } & Nme2a & NDPk_1 $-(153)$ & $\begin{array}{l}n m e 2 a 1 \text { and } n m e 2 a 2 \\
\text { in Salmonids (4R) }\end{array}$ & Eyes and Testis [7, 9], Brain [2] \\
\hline & Nme2b & NDPk_1 $-(153)$ & $\begin{array}{l}\text { Lost in Stickleback } \\
n m e 2 b 1 \text { and } n m e 2 b 2 \\
\text { in Zebrafish (cis-duplication) } \\
\text { and in Salmonids (4R) }\end{array}$ & $\begin{array}{l}\text { nme } 2 b 1 \text { : ubiquitous } \\
\text { (Ovary and Gills) }[1,7,9] \\
\text { (Oocytes) }[3,4,5,6] \\
\text { nme2b2: Muscle }[7,9]\end{array}$ \\
\hline & Nme3 & NDPk_1 - (169) & & $\begin{array}{l}\text { Ubiquitous } \\
\text { (Ovary, Oocyte) }[7,9]\end{array}$ \\
\hline & Nme4 & NDPk_1 $-(190)$ & & $\begin{array}{l}\text { Ubiquitous } \\
\text { (Gonads) }[7,9]\end{array}$ \\
\hline \multirow{3}{*}{$\begin{array}{l}\bar{n} \\
\text { 은 } \\
\text { 는 }\end{array}$} & Nmes & NDPk5 -1217$)$ & & Testis [7, 9] \\
\hline & Nme6 & NDPk6 & & $\begin{array}{l}\text { Ubiquitous } \\
\text { (Ovary, Oocyte and Gills) }[7,9]\end{array}$ \\
\hline & Nme7 & DUF1126 NDPk7A NDPk7B (374) & & $\begin{array}{l}\text { Ubiquitous } \\
\text { (Gonads) }[7,9]\end{array}$ \\
\hline & Nmes & TRX_NDPK NDPK_TX NDPk_TX_Nor_. & & Testis $[7,9]$ \\
\hline & Nme10 & NDPk $-(311)$ & $\begin{array}{l}n m e 10 a \text { and } n m e 10 b \\
\text { in Salmonids (4R) }\end{array}$ & $\begin{array}{l}\text { Ubiquitous } \\
\quad \text { (Ovary, Eyes) }[7,10] \\
\quad \text { (Oocyte) [8, 10] }\end{array}$ \\
\hline
\end{tabular}

1: Lee \& Lee (2000); 2 : Murphy et al (2000); 3 : Bai et al (2007); 4 : Crespel et al (2008); 5 : Keyvanshokook \& Vaziri (2008); 6 ; Ziv et al (2008);

7: Desvignes et al (2009); 8 : Hurd et al (2010); 9 : Desvignes et al (2011); 10 ; Shu et al (2011) 


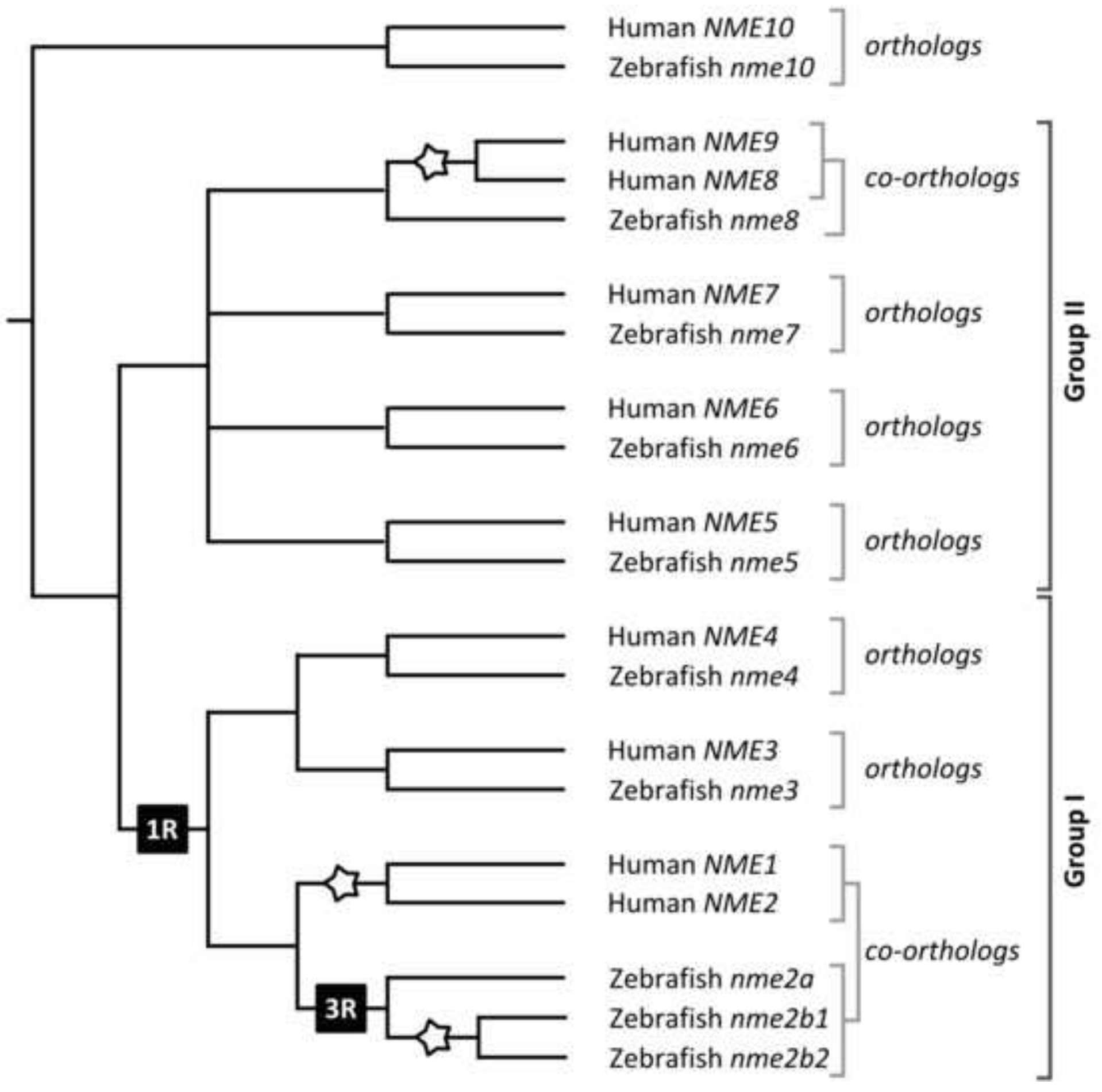

